\title{
Correction to: Impact of Patient and Lesion Characteristics on Drug-Coated Balloon Angioplasty in the Femoropopliteal Artery: A Pooled Analysis of Four Randomized Controlled Multicenter Trials
}

\author{
Thomas Albrecht ${ }^{1,9} \cdot$ Antonia Ukrow ${ }^{1}$ Michael Werk ${ }^{2}$ - Gunnar Tepe ${ }^{3}$. \\ Thomas Zeller $^{4} \cdot$ Dirk-Roelfs Meyer $^{5} \cdot$ Maren Kutschera $^{6} \cdot$ Ulrich Speck $^{6}$. \\ Matthias Waliszewski ${ }^{7,8}$
}

Published online: 12 March 2019

(C) Springer Science+Business Media, LLC, part of Springer Nature and the Cardiovascular and Interventional Radiological Society of Europe (CIRSE) 2019

Correction to: Cardiovasc Intervent Radiol (2019) 42:495-504 https://doi.org/10.1007/s00270-018-2137-3

In their recently published pooled analysis of four drugcoated balloon (DCB) studies, which focused on the impact of patient and lesion characteristics on LLL at 6 months (Albrecht $\mathrm{T}$ et al. Cardiovasc Intervent Radiol. 2018 Dec 11. https://doi.org/10.1007/s00270-018-2137-3), the

The original article can be found online at https://doi.org/10.1007/ s00270-018-2137-3.

Thomas Albrecht

Thomas.albrecht@vivantes.de

1 Department of Radiology and Interventional Therapy, Vivantes Klinikum Neukölln, Berlin, Germany

2 Department of Radiology, Martin-Luther-Hospital, Berlin, Germany

3 Department of Radiology, RoMed Klinikum Rosenheim, Rosenheim, Germany

4 Department of Angiology, Herzzentrum Bad Krozingen, Bad Krozingen, Germany

5 Department of Diagnostic and Interventional Radiology, Hubertus Hospital, Berlin, Germany

6 Experimental Radiology, Department of Radiology, Charité, Berlin, Germany

7 Medical Scientific Affairs, B.Braun Melsungen AG, Berlin, Germany

8 Department of Internal Medicine and Cardiology, Charité Universitätsmedizin Berlin, Campus Virchow Klinikum, Berlin, Germany

9 Institut für Radiologie und Interventionelle Therapie, Vivantes Klinikum Neukölln, Rudower Str. 48, 12351 Berlin, Germany authors reported slightly inaccurate 2-year mortality rates. We would like to rectify this. The discrepancies were as follows: The correct mortality rates are $7.0 \%$ for the control group and $8.6 \%$ for the DCB group $(p=0.55)$, while the authors erroneously reported these rates as $5.5 \%$ (controls) and $7.9 \%$ (DCB, $p=0.32$ ). The reason for these discrepancies was mainly the imprecise calculation of the number of patients available for 2-year follow-up. Details can be found in a follow-up paper published recently in CVIR (Albrecht T, Schnorr B, Kutschera M et al. Cardiovasc Intervent Radiol. 2019. https://doi.org/10.1007/ s00270-019-02194-w).

Publisher's Note Springer Nature remains neutral with regard to jurisdictional claims in published maps and institutional affiliations. 